\title{
Surprise and blocking: Effects of the number of compound trials
}

\author{
N. J. MACKINTOSH \\ Laboratory of Experimental Psychology, University of Sussex, Brighton BN1 9QG, England \\ ANTHONY DICKINSON \\ Department of Experimental Psychology, University of Cambridge, Cambridge CB2 3EB, England
}

and

M. M. COTTON

University of Newcastle, Newcastle, New South Wales, Australia

\begin{abstract}
In an experiment on conditioned suppression of licking in thirsty rats, subjects received either one or two trials to a tone-light compound, either preceded or not preceded by four conditioning trials to the light alone. Prior conditioning to the light blocked conditioning to the tone in subjects receiving two compound trials but not in those receiving only one compound trial. The blocking observed in the two-trial groups was abolished either by the addition of an unexpected second posttrial shock $10 \mathrm{sec}$ after the first on each compound trial or by the omission of an expected second, posttrial shock. The comparable operations had no effect on conditioning to the tone in the one-trial group. In this preparation, both blocking and the attenuation of blocking by surprising changes in reinforcement appear to require more than one trial to appear.
\end{abstract}

In experiments on conditioned suppression in rats, Kamin (1969) established that prior conditioning to one component of a compound conditioned stimulus (CS) could prevent or block conditioning to the other component and that this blocking effect was abolished, or at least attenuated, if a surprising change in reinforcement was programmed to occur on compound trials. In Kamin's original experiments, the change in reinforcement consisted of either an increase in intensity of the shock reinforcer or the addition of an unexpected second, posttrial shock shortly after the first shock reinforcer on each compound trial. A possible interpretation of Kamin's results, therefore, is that blocking occurs because prior conditioning ensures that there is little or no discrepancy between the reinforcement that occurs on compound trials and the reinforcement already expected by the subject on the basis of the pretrained component (Rescorla \& Wagner, 1972). Successful excitatory conditioning to the added component requires such a positive discrepancy between obtained and expected reinforcement, which is provided by an increase in shock intensity or the addition of a second shock.

This research was supported by grants from the United Kingdom Science Research Council. We are grateful to H. Conway for help with running the animals. Requests for reprints should be sent to N. J. Mackintosh, Laboratory of Experimental Psychology, University of Sussex, Brighton BN1 9QG, England.
At least two lines of evidence argue against Rescorla and Wagner's interpretation of Kamin's data. First, it is sometimes difficult to obtain evidence of any blocking at all on the very first compound trial (Mackintosh, 1975b) and equally difficult to observe an effect of a surprising change in reinforcement on this trial (Mackintosh, Bygrave, \& Picton, 1977). Secondly, in multitrial experiments, it is possible to enhance conditioning to the added CS by surprising changes in reinforcement that can hardly be regarded as increasing the positive discrepancy between obtained and expected reinforcement. Thus, both the postponement of an expected posttrial shock (Dickinson, Hall, \& Mackintosh, 1976, Experiments 1 and 2) and its complete omission (Dickinson et al., 1976, Experiment 3; Mackintosh et al., 1977, Experiment 2; Dickinson \& Mackintosh, 1979, Experiment 1) are sufficient to enhance conditioning to the added CS.

The primary aim of the present experiment was to see whether these effects, previously reported in several different experiments, could be obtained in a single study. In particular, it was important to us to know whether a surprising change in reinforcement on compound trials would increase conditioning to the added CS only when conditioning to that CS would otherwise have been blocked. Such a demonstration would provide compelling evidence that surprising changes in reinforcement serve to enhance conditioning to the added CS by attenuating blocking. 
The experiment to be reported was, therefore, a study of conditioned suppression in rats and examined two effects. The first was the effect of prior conditioning to a light CS on subsequent conditioning to a tone CS when the subjects received either one or two reinforced trials with a tone-light compound CS. On the basis of prior research, we should expect to observe blocking only after two compound trials. The second was the effect of introducing surprising changes in reinforcement on compound trials brought about by either the unexpected addition or omission of a second, posttrial shock $10 \mathrm{sec}$ after the first shock reinforcer. If surprising changes in reinforcement serve to enhance conditioning by attenuating blocking, we should also expect to observe such an enhancement only after two compound trials.

Finally, it should be noted that the control group against which we assess the occurrence of blocking differs from that conventionally employed. Most current accounts of blocking (e.g., Kamin, 1969; Mackintosh, 1975a; Rescorla \& Wagner, 1972) argue, in one way or another, that the deficit in conditioning to the added CS arises because it provides no new information about the occurrence of potential reinforcers. Recently, however, Randich and LoLordo (1979) have suggested that the effectiveness of the reinforcer may habituate during initial conditioning to the pretrained $\mathrm{CS}$, thus reducing its ability to support conditioning during compound training in the blocked condition. In order to ensure that any blocking observed in the present experiment was due to the predictive significance of the pretrained CS, rather than to habituation of the reinforcer, the blocked and nonpretrained control groups received equal exposure to shock prior to compound conditioning.

\section{METHOD}

\begin{abstract}
Subjects
The subjects were 144 female hooded Lister rats from the colony maintained at the University of Sussex, aged between 4 and 6 months at the start of the experiment. They were housed, with unlimited access to food, in cages of four. One week before the start of the experiment they were gradually accustomed to a schedule of water deprivation that permitted 30 -min access to water each day. Throughout the experiment, they received their water ration within $15 \mathrm{~min}$ of the end of each training session.
\end{abstract}

\section{Apparatus}

The apparatus consisted of two $20 \times 20 \times 20 \mathrm{~cm}$ chambers with Perspex walls and ceilings and a floor consisting of nine .6-cm-diam stainless steel rods. A $1-\mathrm{cm}$-diam hole, $3 \mathrm{~cm}$ above the floor in the center of one wall, permitted access to a drinking tube connected to a water reservoir; a lick at the tip of the tube opened a springloaded valve and started water flowing. Licks were recorded by a drinkometer when the rat closed a circuit between the tube and a small metal plate surrounding the hole.

Conditioned stimuli consisted of a light provided by switching on a $60-\mathrm{W}, 240-\mathrm{V}$ strip light mounted above the translucent ceiling (light $C S$ ), a $1-\mathrm{Hz}$ flashing light from a $3-\mathrm{W}, 24-\mathrm{V}$ jewel lamp mounted $12 \mathrm{~cm}$ above the drinking tube (flashing lamp CS), and a
78-dB ( re: $20 \mu \mathrm{N} / \mathrm{m}^{2}$ ) $1,800-\mathrm{Hz}$ tone delivered from a speaker mounted $3.5 \mathrm{~cm}$ above the drinking tube. In the absence of the stimuli, the chamber was in complete darkness and a white masking noise was delivered from the speaker. Shocks of $.5 \mathrm{sec}$ duration could be delivered to the grid floors from Campden Instruments shock sources (No. 512). These shock sources give a constant current square-wave output with a $47 \%$ duty cycle. The intensity was set to give a peak output of $.75 \mathrm{~mA}$. The shocks were delivered via Campden Instruments shock scramblers (No. 521s) that run at $25 \mathrm{~Hz}$ with eight separate states per cycle. The chambers were placed inside light- and sound-resistant cubicles.

\section{Procedures}

On Day 1, the rats were placed in the apparatus for $10 \mathrm{~min}$ with the water tube projecting $2 \mathrm{~cm}$ into the chambers. On Days $2-4$, the tips of the tubes were flush with the wall and licks were recorded. Each of these sessions lasted for $610 \mathrm{sec}$ from the time that a rat had completed 200 licks. The 200 th lick started a $10-\mathrm{sec}$ period that was followed by 101 -min intervals, the first and second of which were used in subsequent test sessions for recording licks during the pre-CS and CS periods, respectively.

Conditioning began on Day 5 and was conducted "off-baseline" with the water tubes removed. There was a single conditioning trial during each daily $10-\mathrm{min}$ session. The trial began $3 \mathrm{~min}$ after the start of a session and consisted of the presentation of a 1-min CS terminating in a shock reinforcer. The design of the experiment involved a two-stage blocking procedure and is illustrated in Table 1. There were two conditions, a double-shock condition and a singleshock condition, each comprising three groups. All rats received a compound of the tone and light CSs during each trial in Stage 2. In the double-shock condition, each tone-light compound trial was followed by a second, posttrial shock $10 \mathrm{sec}$ after the first shock reinforcer, while animals in the single-shock condition received only the shock reinforcer. In Stage 1, animals in the blocked and surprise groups received four trials with the light CS. Animals in the blocked groups received the same shock sequence during Stage 1 as that experienced in Stage 2; each light CS terminated with a shock reinforcer, which in the double-shock condition was followed $10 \mathrm{sec}$ later by a posttrial shock. By contrast, animals in the surprise groups experienced a change in the posttrial shocks received from Stages 1 to 2 ; in the double-shock condition, each light trial in Stage 1 ended with only the shock reinforcer; in the single-shock condition, the light in Stage 1 was followed by the two shocks. A comparison of conditioning to the tone in the blocked and the surprise groups would indicate whether a change in posttrial shocks enhanced the level of conditioning to the added tone. In order to assess whether conditioning to the light in Stage 1 did actually block conditioning

Table 1

Design of Experiment

\begin{tabular}{cccccc}
\hline & \multicolumn{2}{c}{ Treatment } & & \multicolumn{2}{c}{ Mean Pre-CS Lick Rate* } \\
\cline { 3 - 4 } Group & Stage 1 & Stage 2 & & One Trial Two Trials \\
\hline Control & L*++ & TL++ & 159 & 155 \\
Blocked & L++ & TL++ & 170 & 174 \\
Surprise & L+ & TL+t & 184 & 170 \\
& & Single-Shock Condition & \\
Control & L*+ $^{*}$ & TL+ & 150 & 164 \\
Blocked & L+ & TL+ & 210 & 206 \\
Surprise & L++ & TL+ & 164 & 138 \\
\hline
\end{tabular}

Note $-L=$ overhead light; $L *$ flashing lamp; $T=$ tone $+=$ single shock immediately after trial; $++=$ double shock with $10-\mathrm{sec}$ intershock interval. Treatment Stage I included four trials; Treatment Stage 2 included one or two trials. $\quad{ }^{*}$ Licks/minute. 
to the tone in Stage 2, control groups were run in both double and single-shock conditions. To control for the effect of prior exposure to shock, these control groups received four trials with the flashing lamp CS in Stage 1. For animals in the double-shock condition these trials ended with two shocks, whereas rats in the single-shock condition received only the shock reinforcer. Twelve animals in each of the six groups received only a single compound trial, while the remaining 12 animals in each group received two compound trials.

Following conditioning, all subjects received two recovery sessions identical to the pretraining sessions on Days 2-4. These were followed by five test sessions, each containing nonreinforced presentation of the tone during the CS period so that suppression conditioned to the tone could be measured during extinction. The number of licks in the pre-CS and CS periods was recorded separately on each trial.

\section{RESULTS}

The results of interest concern the suppression to the tone on test trials. In order to attenuate the effects of individual differences in rate of licking, suppression was expressed as a ratio of the form $A /(A+B)$, where $\mathrm{A}$ was the number of licks during the CS period and $\mathrm{B}$ the number of licks during the pre-CS period. A score of 0 indicates total suppression, whereas one of .50 indicates no suppression.

The mean suppression ratios to the tone averaged over all five test trials are shown separately for the groups receiving only a single compound trial in the left-hand panels of Figure 1 and for the groups receiving two compound trials in the right-hand panels. An overall analysis revealed that the rats receiving a double shock on each trial in Stage 2 were more sup-

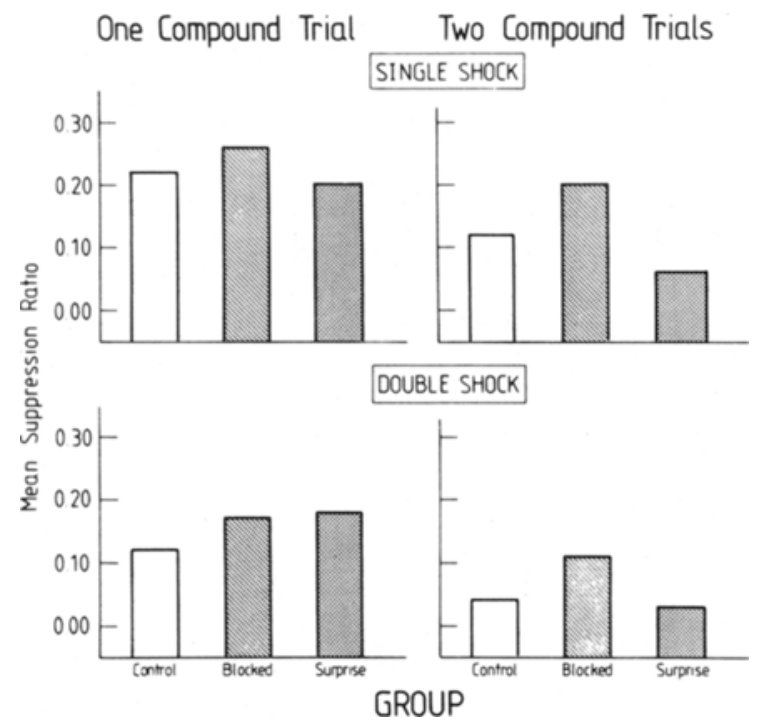

Figure 1. Test suppression ratios of groups receiving either one (left-hand panels) or two compound trials (right-hand panels). The top panels show the data for groups receiving a single shock on each compound trial, and the lower panels show the data for groups receiving two shocks. pressed than those experiencing only a single shock $[\mathrm{F}(1,132)=11.46, \mathrm{p}<.01]$, as were those receiving two rather than one compound trial $[\mathrm{F}(1,132)=23.58$, $\mathrm{p}<.01]$. The interaction between the number of trials and the number of shocks during Stage 2 was not significant $(\mathrm{F}<1)$.

The left-hand panels of Figure 1 show that the treatments received during Stage 1 had no major or systematic effects on conditioning to the tone during the single compound trial of Stage 2 . Both blocked groups, it is true, were slightly less suppressed to the tone than their respective control groups, but the differences were very small, and only one of the two surprise groups showed more suppression than the corresponding blocked groups. An analysis of the test suppression ratios following a single compound trial, using the error term from the overall analysis, revealed neither a significant effect of the treatment in Stage 1 nor a significant interaction between this factor and the number of shocks received in Stage $2(F<1$ in both cases).

By contrast, the right-hand panels of Figure 1 show that for the animals given two compound trials in Stage 2 the blocked groups were less suppressed than their appropriate controls. In addition, the change in reinforcement experienced by the surprise groups appeared to restore the level of conditioning to the tone to at least that of the control groups. In the twocompound trial condition there was a significant effect of the treatment during Stage $1[\mathrm{~F}(2,132)=4.73$, $\mathrm{p}<.01$ ], which did not interact with the number of shocks received during Stage $2(F<1)$. In the absence of a significant interaction, the suppression ratios for the control, blocked, and surprise groups were combined across the single- and double-shock conditions for individual comparisons of the effects of the various treatments in Stage 1. Newman-Keuls tests revealed that the blocked groups differed from both the surprise and the control groups $(p<.05$ in both cases) but that the control and surprise groups did not differ.

Inspection of Figure 1 suggests that the group differences for the rats receiving two compound trials might have been obscured by a "floor" effect, especially in the double-shock condition. Consequently, an a posteriori analysis was conducted on the mean suppression ratios of the rats in the two-trial condition averaged over only the last two test trials. The mean suppression ratios on these two trials for the groups in the single-shock condition were: control, .23; blocked, .32; and surprise, .10; in the double-shock condition, the corresponding ratios were: control, .08; blocked, .26; and surprise, .08. The pattern of group differences was similar to that for the mean suppression ratios averaged over all five test trials. The control and surprise groups in each shock condition exhibited more suppression than the corresponding blocked groups. In addition, the surprise group in the 
single-shock condition appeared to be more suppressed than the control group. An overall analysis of variance revealed significant effects of the number of shocks in Stage $2[F(1,66)=5.60, p<.05]$ and of the treatment in Stage $1[\mathrm{~F}(2,66)=11.94, \mathrm{p}<.01]$, but no significant interaction between these two effects $[\mathrm{F}(2,66)=1.31, \mathrm{p}>.25]$. Individual comparison within each shock condition by the Newman-Keuls procedure showed that, in the single-shock condition, the surprise group was significantly more suppressed than both the control and blocked groups $(\mathrm{p}<.05$ in both cases), which, in turn, did not differ. In the double-shock condition, both the surprise and control groups, which did not differ, were more suppressed than the blocked group ( $p<.05$ in both cases).

The mean pre-CS lick rates for all 12 groups averaged over all five test trials are displayed in Table 1. An overall analysis revealed only a significant effect of Stage 1 treatment $[F(2,132)=3.63, p<.05]$. Subsequent individual comparisons by Newman-Keuls tests within each condition showed that the lick rate of the surprise group in the two-trial, single-shock condition was significantly lower than that of the corresponding blocked group.

\section{DISCUSSION}

The present results confirm and extend those obtained in earlier experiments. In this preparation, at least, there is no evidence of blocking or of any effect of a surprising change in reinforcement on the first compound trial. A second compound trial, however, is sufficient to produce a reliable blocking effect, and, once blocking occurs, it is also possible to see the effects of a surprising change in reinforcement. Finally, it is immaterial whether that change consists in the addition of an unexpected second shock or in omission of an expected one.

This pattern of results had significant implications for analyses of blocking. First, if blocking does not occur on the first compound trial, it can hardly be a consequence of any limitation of the effectiveness of predicted reinforcers, as Rescorla and Wagner (1972) imply. It is not, of course, possible to prove the null hypothesis, and it is true that blocking can be obtained in flavor-aversion after a single trial (Gillan \& Domjan, 1977). Nevertheless, the present study employed large numbers of subjects and was sufficiently sensitive to detect reliable blocking after only two compound trials. These results, therefore, reinforce our contention that, in this preparation, blocking is not readily detected on the first compound trial. Furthermore, the blocking effect observed in this study cannot be due to habituation to the shock, for the blocked and control groups had the same exposure to shock during the first stage. It should be noted, however, that the use of this control group may have reduced the magnitude of the blocking effect observed by generalization between the flashing and overhead light CSs. Second, the role of surprising changes in posttrial stimulation cannot be to reinforce conditioning to the added CS, because such an effect should be demonstrable after only a single compound trial and should also be confined to the double-shock condition, in which the change produces a potential increase in the effective magnitude of reinforcement.

These results are much more readily explained by those accounts of blocking which suggest that there is a decline in the associability of the added CS after subjects have been exposed to it in a context in which the occurrence of the reinforcer and any other posttrial event are otherwise predicted. Such a decline in the associability of the added CS in the blocked group after the first compound trial will have no effect on conditioning on that trial but will serve to attenuate conditioning to this CS on subsequent compound trials (Mackintosh, 1975a; Wagner, 1978). The effect of surprising changes in posttrial stimulation must be to interfere with the process responsible for this decline in the associability of the added CS, either by insuring that this CS does predict some events of consequence (Dickinson \& Mackintosh, 1979) or by disrupting the process of habituation which might underlie the decline (Wagner, 1978).

It is notable that an analysis of the last two test trials in the two-compound-trial condition revealed that the surprise group in the single-shock condition was suppressed not only more than the blocked group, but also more than the appropriate control group. If we accept this result at face value (and it should be remembered that this surprise group had an unusually low pre-CS rate), it implies that the unexpected omission of a posttrial shock may not merely eliminate the blocking effect, but may actually result in more conditioning to the added CS than in a control group. The possibility that such an enhancement will be observed when posttrial surprise is generated by the omission of an expected shock can, in fact, be explained by Mackintosh's (1975a) theory of associative learning (see Dickinson \& Mackintosh, 1979, p. 175). According to this theory, the associability of a CS will increase when that CS is a better predictor than are other stimuli present of the occurrence or nonoccurrence of the reinforcer and posttrial stimuli. In the singleshock condition, the pretrained CS is established in Stage 1 as a predictor of the posttrial shock in the surprise group, but not in the control group. Consequently, in the surprise, but not in the control, group, the added CS will be a better predictor of the omission of the posttrial shock on the first compound trial than the pretrained CS. As a result, there will be a factor tending to enhance the associability of the added CS 
above its starting value after the first compound trial in the surprise group, which, in turn, may lead to greater conditioning on the second compound trial than that seen in the control group. Such a factor will not operate in the double-shock condition, in which the added and pretrained CSs start as equivalent predictors of the posttrial shock on the initial compound trials in both the surprise and control groups.

\section{REFERENCES}

Dickinson, A., Hall, G., \& Mackintosh, N. J. Surprise and the attenuation of blocking. Journal of Experimental Psychology: Animal Behavior Processes, 1976, 2, 313-322.

Dickinson, A., \& Mackintosh, N. J. Reinforcer specificity in the enhancement of conditioning by posttrial surprise. Journal of Experimental Psychology: Animal Behavior Processes, 1979, $5,162-177$.

Gillan, D. J., \& DomJan, M. Taste aversion conditioning with expected versus unexpected drug treatment. Journal of Experimental Psychology: Animal Behavior Processes, 1977, 3, 297-309.

Kamin, L. J. Predictability, surprise, attention and conditioning. In B. Campbell \& R. Church (Eds.), Punishment and aversive behavior. New York: Appleton-Century-Crofts, 1969.
MaCkintosh, N. J. A theory of attention: Variations in the associability of stimuli with reinforcement. Psychological Review, $1975,82,276-298$. (a)

MaCkintosh, N. J. Blocking of conditioned suppression: Role of the first compound trial. Journal of Experimental Psychology: Animal Behavior Processes, 1975, 1, 335-345. (b)

Mackintosh, N. J., Bygrave, D. J., \& Picton, B. M. B. Locus of the ef fect of a surprising reinforcer in the attenuation of blocking. Quarterly Journal of Experimental Psychology, $1977,29,327-336$.

Randich, A., \& LoLordo, V. M. Associative and nonassociative theories of the UCS preexposure phenomenon: Implications for Pavlovian conditioning. Psychological Bulletin, 1979, 86, 523-548.

Rescorla, R. A., \& Wagner, A. R. A theory of Pavlovian conditioning: Variations in the effectiveness of reinforcement and nonreinforcement. In A. H. Black \& W. F. Prokasy (Eds.), Classical conditioning II: Current research and theory. New York: Appleton-Century-Crofts, 1972.

WAGNER, A. R. Expectancies and the priming of STM. In S. H. Hulse, H. Fowler, \& W. K. Honig (Eds.), Cognitive processes in animal behavior. Hillsdale, N.J: Erlbaum, 1978.

(Received for publication October 30, 1979; revision accepted February 15, 1980.) 